\title{
Mechanism of Increased Alpha Adrenergic Vasoconstriction in Human Essential Hypertension
}

\author{
Brent Egan, Roberto Panis, Alan Hinderliter, Nik Schork, and Stevo Julius \\ Division of Hypertension, Department of Internal Medicine, University of Michigan, Ann Arbor, Michigan 48109-0356
}

\begin{abstract}
Multiple components of vascular alpha adrenergic responsiveness were investigated in twenty-four men with mild hypertension and eighteen age- and weight-matched normotensive controls. Arterial plasma norepinephrine (paNE), an index of sympathetic drive, was increased in hypertensives compared to normotensives (mean \pm SE), 199 \pm 24 vs. $134 \pm 11 \mathrm{pg} / \mathrm{ml}, P$ $<0.02$. The effective concentration of intra-arterial (iaNE) increasing forearm vascular resistance (FAVR) 30\% (NE-EC30, an index of vascular alpha-receptor sensitivity) was similar in normotensives and hypertensives, $9 \pm 1 \mathrm{vs} .13 \pm 3 \mathrm{ng} / 100 \mathrm{ml}$ per min, respectively, $P>0.3$. The phentolamine induced reduction in FAVR, an index of vascular alpha-tone, was greater in hypertensives, $-21.3 \pm 1.8$ vs. normotensives, $-14.9 \pm 1.2 \mathrm{U}, P$ $<0.02$.

We interpret these data as evidence for normal vascular alpha-receptor sensitivity to norepinephrine in mild hypertensives. Consequently, the increased sympathetic drive in mild hypertensives explains the elevated vascular alpha-tone. Although vascular alpha-receptor sensitivity to iaNE was normal, the FAVR responses at high doses (reactivity) were greater in hypertensives to regional infusion of both NE and angiotensin II. This "nonspecific" enhancement of vascular reactivity is probably explained by structural vascular changes in hypertensives.
\end{abstract}

\section{Introduction}

Increased total peripheral resistance (TPR) ${ }^{1}$ is the pathophysiologic hallmark of human essential hypertension (EHT) (1). Previous studies showed that enhanced alpha-adrenergic vasoconstriction contributes to increased TPR in EHT. More specifically, investigations of regional vascular responses identified increased vasodilation to alpha-receptor antagonists (2) and greater vasoconstriction to alpha-agonists $(3,4)$ in hypertensives compared to normotensives. Similarly, greater pressor responses to intravenous infusions of norepinephrine were observed in hypertensive subjects $(5,6)$. In a subset of hyperten-

\section{Address reprint requests to Dr. Egan. \\ Received for publication 3 October 1986 and in revised form 27 February 1987.}

1. Abbreviations used in this paper: EHT, essential hypertension; FABF, forearm blood flow; iaAII, intraarterial angiotensin II; iaNE, intraarterial norepinephrine; $\mathrm{mFAVR}$, minimum forearm vascular resistance; MAP, mean arterial pressure; PRA, plasma renin activity; TPR, total peripheral resistance.

J. Clin. Invest.

(C) The American Society for Clinical Investigation, Inc.

0021-9738/87/09/0812/06 \$2.00

Volume 80, September 1987, 812-817 sive patients with high-renin and increased plasma norepinephrine, arterial pressure was normalized when autonomic cardiac blockade was followed by phentolamine $(7,8)$.

Although these studies each confirmed a role for the sympathetic system in hypertension, the apparent mechanism of the adrenergic contribution differed. The sympathetic "lesions" identified included increased sympathetic drive (7), specifically increased alpha-receptor sensitivity $(2,5)$, nonspecifically increased responses to norepinephrine attributed to either structural reinforcement $(9,10)$ or abnormal cellular cation metabolism (11). None of the studies simultaneously evaluated multiple potential explanations for enhanced alphaadrenergic vasoconstriction in EHT. The present investigation is the first to investigate each of the possible alpha-adrenergic abnormalities in a group of hypertensive patients and matched normotensive controls.

In this study, arterial plasma norepinephrine served as an index of sympathetic drive, forearm vasodilation to phentolamine was the index of vascular alpha-tone, and forearm vasoconstriction to intraarterial norepinephrine (iaNE) was used to determine vascular alpha-receptor sensitivity. Vasoconstrictor responses to intra-arterial angiotensin II (iaAII) acted as a control for the specificity of responsiveness to iaNE. Five established criteria for indirectly assessing the influence of vascular structure on resistance responses were examined $(9,10)$.

\section{Methods}

Subjects. 24 patients with mild hypertension and eighteen age- and weight-matched normotensive volunteers were studied after a minimum of 3 wk off all medication. Subjects were recruited by advertisement and paid. All were men in good health between 27 and 54 yr. Each read and signed an approved informed consent before participation.

Measurements: Physiologic. Arterial pressure was measured through a 20-gauge, 2-inch plastic catheter in the left brachial artery. The cannula was connected by an arterial pressure monitoring line (American Pharmaseal, Valencia, CA) to a Hewlett-Packard (Andover, MA) 1290A quartz transducer, $4568 \mathrm{C}$ polygraph, 1308A oscilloscope, and Gould (Cleveland, OH) TA-600 thermal recorder. Mean arterial pressure (MAP, in millimeters of mercury) was determined by the electronically integrated area under the brachial arterial pulse-wave form.

Forearm volume was measured by water displacement. Forearm blood flow (FABF) was measured by mercury-in-silastic strain-gauge, venous occlusion plethysmography. The left forearm was supported at the wrist and elbow above heart level. The strain gauge encircled the forearm $\sim 7 \mathrm{~cm}$ distal to the olecranon. $1 \mathrm{~min}$ before FABF measurement, a pediatric cuff was inflated at the wrist to suprasystolic pressure to occlude hand blood flow. The venous congesting cuff on the upper arm was inflated to $\mathbf{4 0 - 5 0 ~} \mathrm{mmHg}$ for $10-15 \mathrm{~s}$ and deflated for 3-5 s over four cycles with the Hokanson (Issaquah, WA) E-10 rapid cuff inflator. The strain gauge was connected to a Hokanson EC-4 plethysmograph. FABF in milliliters per $100 \mathrm{ml}$ forearm volume/min was determined from the mean vertical deflection/minute obtained on the 
four tracings divided by the $1 \%$ electrical calibration signal. Forearm vascular resistance (FAVR, arbitrary units) was calculated as MAP/ FABF.

Minimum forearm vascular resistance (mFAVR, arbitrary units) was calculated by dividing MAP by maximal FABF. Maximal FABF was determined as the flow achieved after $10 \mathrm{~min}$ of ischemic forearm exercise. The upper congesting cuff was inflated to suprasystolic pressure for $10 \mathrm{~min}$. During this interval the subject contracted the hand for $5 \mathrm{~s}$ of each 30-s interval. The maximal flow was calculated from the six peak flow curves obtained in the $60-90 \mathrm{~s}$ immediately after ischemia. A larger number of tracings was obtained as the inflation-deflation cycle lasted 6-8 s under peak flow conditions. Previous work with the mFAVR measurement in 10 normotensive volunteers in our laboratory found intra- and inter-day coefficient of variation of 8 and $11 \%$, respectively.

Biochemical measurements. Plasma norepinephrine and epinephrine concentrations were determined on plasma obtained from arterial blood by the single isotope radioenzymatic assay described by Peuler and Johnson (12). Plasma renin activity (PRA) was measured by radioimmunoassay of generated angiotensin I by the method of Haber et al. (13).

Protocol. Subjects came to the laboratory at 0800 hours. Height, weight, forearm volume and casual (seated) blood pressure were measured. Once supine, the left forearm was prepared under sterile conditions, and a 20-gauge, 2-in. plastic cannula was inserted percutaneously into the left brachial artery. 30 min after catheter placement, measurements of FABF were obtained. After three consecutive series of FABF measurements were within a range of $\pm 10 \%$, baseline supine arterial blood was obtained for plasma catecholamine and renin determinations. Room temperature was controlled in the range of 77 to 81 degrees Fahrenheit in order to obtain stable FABF. Nevertheless, two normotensive and three hypertensive individuals not included in the total of 42 subjects were excluded because of variability in baseline FABF.

After the baseline, norepinephrine (NE) was infused in six sequential ascending doses into the brachial artery (ia). The doses were $0.00125,0.005,0.02,0.08,0.16,0.24 \mu \mathrm{g} / 100 \mathrm{ml}$ forearm volume/ minute. After a second baseline period to reestablish stable FABF, angiotensin II (AII) was infused in six doses of 0.125, 0.5, 2.0, 8.0, 16.0, $24.0 \mathrm{ng} / 100 \mathrm{ml}$ forearm volume/minute. Each infusion of NE and AII lasted 4 min with measurement of MAP and FABF during the fourth minute. In a pilot study in 13 normotensive volunteers, the FAVR levels generated by the sequential infusion and a random order infusion of iaNE with intervening 11-min baselines were similar (unpublished data). This indicated an absence of tachyphylaxis during the duration of the sequential infusion that was chosen for this study to save time. After a third stable baseline FABF was obtained, phentol- amine was infused for $10 \mathrm{~min}$ at $12 \mu \mathrm{g} / 100 \mathrm{ml}$ forearm volume/ minute.

Statistical analysis. Descriptive data are reported as mean \pm SE. Differences between hypertensives and normotensives for the descriptive variables were evaluated by Student's two-tailed $t$ test. To evaluate the dose-response curves to iaNE and iaAII, a three-factor ANOVA (14) was used to assess group, drug, and dose effects, or any interaction of these factors.

The dose-response curve for each individual was linearized by logit transformation (14). The concentration of iaNE producing $30 \%$ of the maximum obtained FAVR (NE-EC30) was calculated for each individual. A Student's $t$ test was used to test for group differences in EC30. The repeated measures test was used to compare responses at single points on the dose-response curves in hypertensives and normotensives. Possible relationships of sympathetic drive (plasma norepinephrine), mFAVR, and blood pressure to the FAVR responses were assessed by linear regression analysis.

\section{Results}

Descriptive variables for the hypertensive (HT) and normotensive (NT) groups are enumerated in Table I. The hypertensives were slightly, albeit insignificantly, older. Both groups were well matched for height, weight, and forearm volume. Initial baseline forearm blood flow (FABF) pre-iaNE was also similar in both groups. Casual systolic and diastolic blood pressures measured in the laboratory were higher in hypertensives as expected. Baseline arterial plasma norepinephrine was higher in the hypertensives, while epinephrine and plasma renin activity were similar in the two groups. Despite higher mean arterial pressure in the hypertensives (MAP, 94.4 \pm 3.2 vs. $112.5 \pm 2.5, P<0.0001$ ), their maximal forearm blood flow $(\mathrm{NT}=52.2 \pm 3.1$ vs. $\mathrm{HT}=52.9 \pm 2.9, P=\mathrm{NS})$ was nearly identical to normotensives. Consequently, minimum forearm vascular resistance (mFAVR) was higher in the hypertensive group as shown in Fig. 1.

The dose-response curve to iaNE in hypertensives and normotensives is shown in Fig. 2. Hypertensives had a FAVR response to iaNE which differed from the response of normotensive controls, $P<0.001$ by ANOVA. The hypertensives also achieved higher levels of FAVR at the highest iaNE and iaAII dose, $P<0.05$ by the repeated measures test. Both the absolute FAVR and change in FAVR levels obtained at most of the iaNE and iaAII doses correlated with mFAVR (Table

Table I. Baseline Comparative Data on the Hypertensive and Normotensive Groups

\begin{tabular}{|c|c|c|c|}
\hline & Normotensive & Hypertensive & $P$ \\
\hline & $n=18$ & $n=24$ & \\
\hline Age, $y r$ & $33 \pm 2$ & $37 \pm 2$ & NS \\
\hline Height $\mathrm{cm}$ & $181 \pm 2$ & $178 \pm 2$ & NS \\
\hline Weight $l b s$ & $206 \pm 8$ & $212 \pm 12$ & NS \\
\hline Forearm volume $m l$ & $1,316 \pm 52$ & $1,333 \pm 49$ & NS \\
\hline Forearm blood flow, $\mathrm{ml} / 100 \mathrm{ml} / \mathrm{min}$ & $4.09 \pm 0.34$ & $4.33 \pm 0.33$ & NS \\
\hline Systolic BP, $\mathrm{mmHg}$ & $122 \pm 3$ & $145 \pm 3$ & 0.0001 \\
\hline Diastolic BP, $m m H g$ & $78 \pm 2$ & $100 \pm 2$ & 0.0001 \\
\hline Plasma NE, $p g / m l$ & $134 \pm 11$ & $199 \pm 24$ & 0.02 \\
\hline PRA, $n g A I / h$ & $1.79 \pm 0.32$ & $1.75 \pm 0.23$ & NS \\
\hline Plasma Epi, $p g / \mu l$ & $74 \pm 6$ & $81 \pm 8$ & NS \\
\hline
\end{tabular}




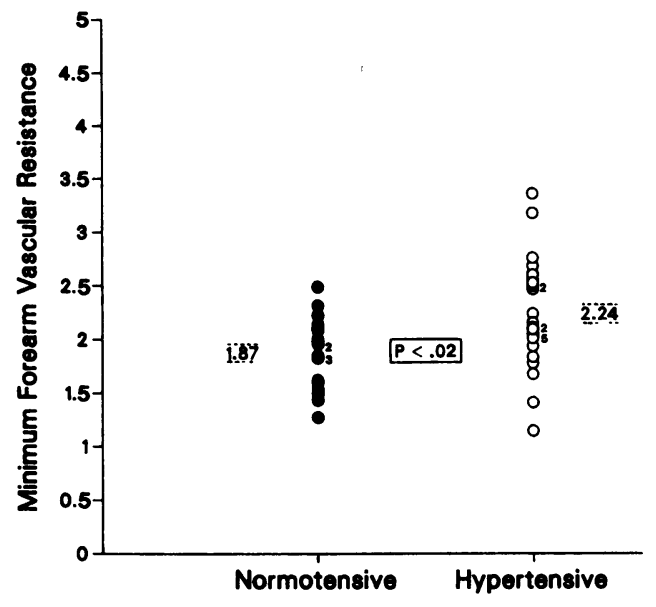

Figure 1. mFAVR obtained after $10 \mathrm{~min}$ of ischemic exercise was significantly higher in hypertensives, shown on the right, as compared with normotensives.

II). While the overall response pattern was different in hypertensives, the effective concentration of iaNE producing $30 \%$ of the maximum obtained change in FAVR (NE-EC30) was similar in normotensives, $9 \pm 1$, and hypertensives, $13 \pm 3 \mathrm{ng} / 100$ $\mathrm{ml}$ per $\mathrm{min}, P=\mathrm{NS}$. The NE-EC30 did not correlate significantly with plasma norepinephrine concentration, $r=0.12, P$ $>0.4$. The FAVR response to iaAII, shown in Fig. 3, was similar to that in response to iaNE. In fact, by ANOVA, the within group response to the two vasoconstrictors was not different. The MAP levels were relatively constant during both infusions as shown in Figs. 2 and 3, although small (2-3 $\mathrm{mmHg}$ ) but significant increases were noted at the highest doses.

Three-factor ANOVA (14) was utilized to evaluate the effects of drug (iaNE vs. iaAII), dose of drug, and group diag-

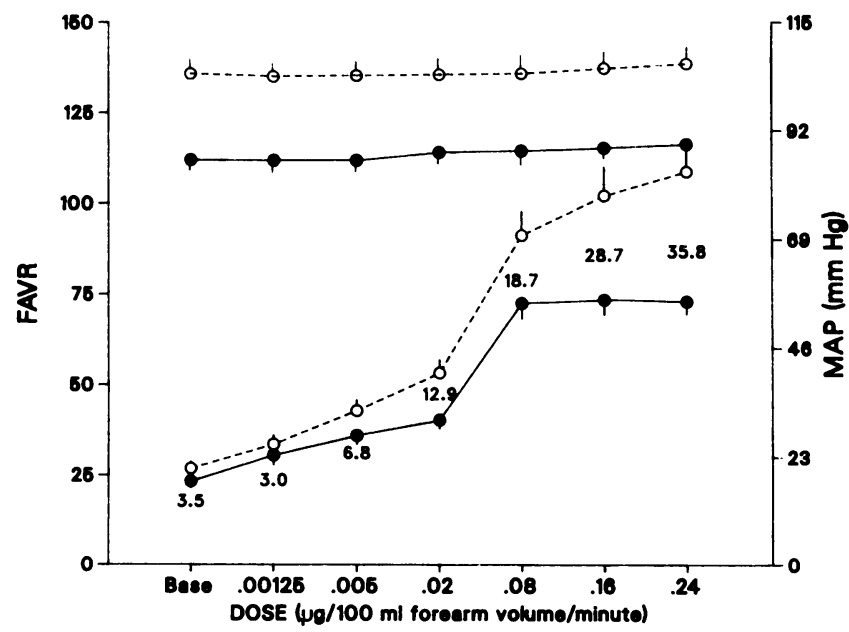

Figure 2. The FAVR to norepinephrine and MAP, $\mathrm{mmHg}$ at baseline and in response to a sequential iaNE infusion are shown separately for hypertensives (open circles, dashed lines) and normotensives (solid circles and lines). The FAVR response curves comparing hypertensives vs. normotensives were different at $P<0.001$ by ANOVA. While the MAP increased slightly at the higher dose, the overall MAP during the infusion was parallel in the two groups. Numbers adjacent to dose-response line represent mean differences in FAVR between the two groups.
Table II. Correlation Coefficients of mFAVR Vs. Absolute FAVR and Change in FAVR in Response to Intraarterial NE and AII

\begin{tabular}{llllll}
\hline Dose ia NE & FAVR & $\Delta$ FAVR & Dose ia AII & FAVR & $\Delta$ FAVR \\
\hline$\mu g / 100 \mathrm{ml} / \mathrm{mm}$ & $n=42$ & $n=42$ & $n g / 100 \mathrm{ml} / \mathrm{liter}$ & $n=41$ & $n=41$ \\
0.00125 & 0.36 & 0.23 & 0.125 & 0.52 & 0.46 \\
0.005 & 0.38 & 0.23 & 0.5 & 0.57 & 0.59 \\
0.02 & 0.53 & 0.46 & 2.0 & 0.49 & 0.34 \\
0.08 & 0.53 & 0.49 & 8.0 & 0.51 & 0.46 \\
0.16 & 0.66 & 0.65 & 16.0 & 0.51 & 0.53 \\
0.24 & 0.69 & 0.65 & 24.0 & 0.53 & 0.48 \\
\hline
\end{tabular}

$r \geq 0.26, P<0.05 ; r \geq 0.37, P<0.01 ; r \geq 0.46, P<0.001$.

nosis (HT vs. NT) singly and in combination on FAVR responses. This analysis showed that response to the two drugs was similar within each group as noted above, $F=0.3, P=$ 0.56 . The other interaction of interest was dose-group which indicates if diagnosis differentially affects response patterns to the intraarterial infusions. This analysis identified differences in group response patterns as a function of dose, $F=8.3, P<$ 0.001 , indicating a steeper dose-response relationship in hypertensives.

The hypertensive group, which manifested a higher baseline FAVR before phentolamine $(31.6 \pm 2.5$ vs. $25.0 \pm 1.7, P$ $<0.04$ ), experienced a significantly greater reduction in FAVR after $10 \mathrm{~min}$ of phentolamine, $-21.3 \pm 1.9$ vs. $-14.9 \pm 1.1, P$ $<0.02$. After phentolamine, both groups had similar FAVR $(\mathrm{HT}=10.3 \pm 1.7, \mathrm{NT}=9.6 \pm 1.0, P=0.75)$. In hypertensives, the percentage reduction in FAVR following the 10 -min phentolamine infusion correlated negatively with arterial plasma norepinephrine, $r=-0.44$, respectively, $P<0.03$. This relationship was not significant in normotensives $r=-0.23, P$ $>0.20$. The increase in FABF after phentolamine correlated positively with the NE-EC30 in hypertensives, $r=0.44, P$ $<0.03$, but not normotensives, $r=-0.13, P>0.5$.

\section{Discussion}

The major objective of this study was to determine if hypertensive subjects had specific abnormalities in adrenergic func-

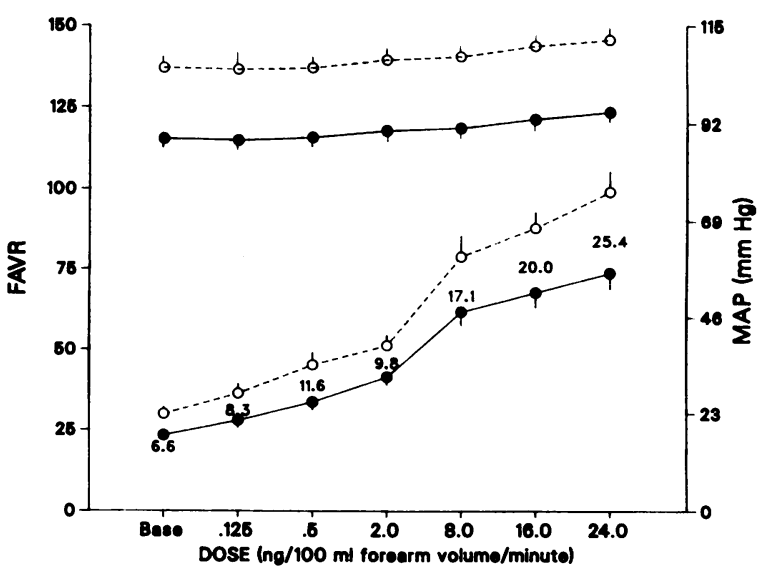

Figure 3. Same as Fig. 2 except that the regionally infused vasoconstrictor is AII. By ANOVA, the dose-response curves were different at $P<0.01$. $\bullet$, Normotensives; $\mathrm{O}$, hypotensives. 
tion contributing to elevated peripheral vascular resistance. The various aspects of the adrenergic system evaluated included sympathetic drive, alpha-receptor sensitivity, vascular reactivity over a wide range of norepinephrine doses, specificity of vascular response pattern to norepinephrine compared to AII control, and vascular alpha-tone. In addition, the influence of vascular structure on reactivity was evaluated indirectly according to established criteria $(9,10)$.

We studied FAVR responses to regional infusion of vasoactive drugs. Regional infusions were chosen to examine a wider range of responses than could be obtained by systemic infusion, which, for safety reasons, are limited by changes in arterial pressure. Since large variations in arterial pressure were avoided, arterial baroreflexes, which are impaired in hypertensives (15), played little role in the observed results.

Arterial plasma norepinephrine, an index of sympathetic drive (16-18) obtained after 30 min supine rest, was elevated in the hypertensives. This finding agrees with a number of prior investigations in young patients with mild disease (19). Additional evidence indicates that the increased plasma noradrenaline in this group of patients reflects increased sympathetic nerve activity (20) with greater spillover from the synaptic cleft. Although, reduced clearance reflecting decreased neuronal reuptake has been described in hypertensives (21), this occurs predominantly in patients older than those in the present study.

The NE-EC30, an index of vascular alpha-receptor sensitivity, was not different in normotensives and hypertensives. This suggests alpha-receptor sensitivity is normal in these mild hypertensives. Ideally, alpha-receptor sensitivity in the two groups is determined under conditions of similar sympathetic drive. Since sympathetic drive (plasma norepinephrine) was elevated in the hypertensive subset, a compensatory reduction or down-regulation in alpha-receptor sensitivity might be predicted. Consequently, one could argue that a normal NE-EC30 in the setting of increased sympathetic drive represents an underlying enhancement of alpha-receptor sensitivity. This is unlikely for two reasons. First, in this study where local vascular responses were evaluated, the NE-EC30 was not related to plasma norepinephrine concentration, $r=0.12, P>0.4$. Second, in another study we found that chronic pharmacologic reduction in plasma norepinephrine, within a group of hypertensives by a percentage similar to the group differences observed in this study, was not associated with enhanced vasoconstriction to iaNE (22).

Normal vascular alpha-receptor sensitivity in the present study is in agreement with the findings of Sivertsson and Olander who conducted a similar study in patients with mild pressure elevation (4). However, these data are discordant with several other reports $(2,3,5)$. Technical factors may explain the differences. For instance, a greater pressor response to intravenous norepinephrine in hypertensives does not necessarily indicate increased alpha-receptor sensitivity. Variable cardiac and baroreflex responses to the elevated pressure may confound analysis of vascular alpha-receptor mediated vasoconstriction. Vasodilation to a maximal regional infusion of an alpha-1 blocker assesses vascular alpha-1 receptor tone. Consequently, it is difficult to extrapolate from this index of alpha-tone to alpha-receptor sensitivity.

A previous study of FAVR responses to regional iaNE reported increased alpha-receptor sensitivity (3). Their results were analyzed as percentage changes in forearm blood flow. We reanalyzed our results by this criterion and did not find any evidence for increased vascular alpha-receptor sensitivity. Since baseline FABF was not reported, it is possible that a lower initial flow in hypertensives, which would produce a higher effective concentration of norepinephrine, caused an apparent increase in alpha sensitivity. It is also conceivable that alpha-receptor sensitivity increases as hypertension becomes more severe (5). If true, that could explain the concordance of our results with one (4) but not the other study (3) of regional vascular alpha sensitivity. The mechanism whereby more severe hypertension could increase alpha-receptor sensitivity is unknown but might involve pressure-induced endothelial dysfunction (23) with relative enhancement of alpha-2 receptor mediated vasoconstriction (24-26).

The response pattern of FAVR to a sequential infusion of norepinephrine served as an index of vascular alpha adrenergic reactivity. Hypertensive subjects, while responding normally at the lowest iaNE dose, diverged progressively from normotensives at higher iaNE doses. Overall, the response patterns to iaNE in normotensives and hypertensives were different by ANOVA, $P<0.001$. However, enhanced vascular reactivity to iaNE in hypertensives was nonspecific, since a similar pattern was seen in response to AII (Fig. 3).

Comparing the overall FAVR responses in hypertensives versus normotensives in this study, we find support for each of the five predicted criteria for vascular structure as the major determinant of response pattern $(9,10)$. (a) As discussed, alpha-receptor sensitivity was normal. An altered vascular structure would not be expected to selectively enhance receptor sensitivity. (b) Beginning with a reduced luminal area, a steeper slope of the vasoconstrictor dose versus vascular resistance response is predicted with equivalent degrees of vasoconstriction, since resistance rises with the fourth power of the radius. By a three-factor ANOVA, evidence was obtained indicating a steeper dose-response relationship in the hypertensives. (c) A structural reduction in vascular cross-sectional area would also contribute to a greater resistance at maximum vascular contraction. The hypertensive subjects generated significantly greater FAVR at the highest dose of NE and AII by the repeated measures test. While it might be argued that maximum vasoconstriction to iaNE was not achieved in hypertensives, the normotensives plateaued at the highest three doses (Fig. 2). (d) A structural abnormality in hypertensives should provide a nonspecific and stereotyped enhanced responsiveness to all vasoconstrictors. In support of this point, the response patterns to iaNE and iaAII were similar by inspection and were not different by ANOVA. (e) Elevated resistance at maximum vasodilation is strong evidence in favor of a structural reduction in cross-sectional area of the resistance vessels. The mFAVR was, in fact, increased in the hypertensives. Furthermore, the resistance values generated in response to the iaNE and iaAII correlated positively with this index of structure. Consequently, each of the five expected criteria supporting vascular structure as a principal determinant of vascular resistance responses was confirmed in this study.

To corroborate the contention that vascular cross-sectional area is structurally diminished in hypertensives, it is necessary to insure maximal vasodilation occurs after $10 \mathrm{~min}$ of ischemic exercise. Supporting evidence includes $(a)$ similar minimal resistances are achieved with 8 and $10 \mathrm{~min}$ of ischemia 
(27). Thus, it is unlikely that a longer period of ischemia would lower resistance substantially more. (b) Resistance after 10 min of ischemic forearm exercise is not raised by lower body negative pressure that normally causes a reflex neurogenic increase in FAVR (28). Thus, the forearm vessels do not respond to endogenous norepinephrine release with vasoconstriction. (c) Systemic infusion of norepinephrine which increased mean blood pressure by $50 \%$ raised flow proportionately and did not raise the mFAVR (27). Consequently, the forearm vasculature after $10 \mathrm{~min}$ of ischemia did not constrict in response to exogenous norepinephrine. $(d)$ Ischemia alone and ischemia plus adenosine, a potent vasodilator, produced similar levels of mFAVR (27). (e) Vasodilation in response to maximum regional infusion of verapamil produces similar levels of maximum flow compared to ischemia in mild hypertensives (29). This indicates that a very potent vasodilator does not lower resistance more than ischemia in this group of patients. $(f)$ The mFAVR is reproducible in our laboratory and others (27, 28 ) indicating the absence of a significant tonic (variable) component. (g) Morphological studies of human vessels support the indirect criteria cited indicating that a structural abnormality is present in hypertensive patients (30-33).

While our data strongly implicate a structural reduction in luminal cross-sectional area as an important contributor to resistance responses, the nature of the vascular abnormality is not proven. The possibilities include increased wall thickness encroaching on the luminal area (increased wall-to-lumen ratio), a reduction in vessel size, or a reduction in vessel number $(9,10,30-33)$. The latter two possibilities need not involve an altered wall:lumen ratio. Since we have not anatomically examined vessel structure we cannot definitively exclude any of the three.

Vascular alpha-tone, assessed by the phentolamine induced decrease in FAVR, was greater in hypertensives. The observation of enhanced vascular alpha-adrenergic vasoconstriction in hypertensives agrees with previous reports $(2,7)$. Increased sympathetic drive was the probable cause of the enhanced alpha-tone in our subjects, since baseline arterial plasma norepinephrine was elevated and sensitivity to iaNE (NE-EC30) was normal. Of note, the phentolamine induced increase in forearm blood flow correlated positively, $r=0.44$, $P<0.05$, with the NE-EC30 in hypertensives only. In other words, the hypertensive patients with the highest vascular alpha-tone displayed the lowest alpha-receptor sensitivity to iaNE. Furthermore, the percentage reduction in resistance after phentolamine correlated inversely and significantly with plasma norepinephrine only in hypertensives. The last two observations are consistent with the view that the increased sympathetic drive and not enhanced alpha-receptor sensitivity explained the increased vascular alpha-tone.

In summary, abnormalities in adrenergic function were identified in a group of relatively young, overweight, mild hypertensives compared with a well-matched normotensive control group. Plasma norepinephrine and the vasodilator response to phentolamine were greater in hypertensives, while vascular sensitivity to norepinephrine was normal. Despite the normal sensitivity to iaNE, vascular reactivity to a graded iaNE infusion was enhanced in hypertensives. A similar FAVR response pattern was observed to iaAII. For several reasons a structural reduction in vascular luminal area likely explains the altered resistance response in hypertensives.
We conclude that increased sympathetic drive was the major reason for the increased vascular alpha-adrenergic tone in this group of hypertensives. Structural vascular changes in these mild hypertensive patients may contribute nonspecifically to further enhance alpha-adrenergic vasoconstriction.

\section{Acknowledgments}

Dr. Egan is the recipient of a National Institutes of Health Clinical Investigator Award, HL-01353. This research was also supported by a grant-in-aid from the American Heart Association of Michigan. Angiotensin II was provided as a gift from Ciba-Geigy.

\section{References}

1. Freis, E. D. 1960. Hemodynamics of Hypertension. Physiol. Rev. 40:27-54.

2. Amann, F. W., P. Bolli, W. Kiowski, and F. Buhler. 1981. Enhanced alpha-adrenoreceptor-mediated vasoconstriction in essential hypertension. Hypertension. 3(Suppl. I):I-1 19-I-123.

3. Doyle, A. E., J. R. E. Fraser, and R. J. Marshall. 1959. Reactivity of forearm vessels to vasoconstrictor substances in hypertensive and normotensive subjects. Clin. Sci. 18:441-453.

4. Sivertsson, R., and R. Olander. 1968. Aspects of the nature of the increased vascular resistance and increased "reactivity" to noradrenaline in hypertensive subjects. Life Sci. 7(Part I):1291-1297.

5. Philipp, T. H., A. Distler, and U. Cordes. 1978. Sympathetic nervous system and blood-pressure control in essential hypertension. Lancet. ii:959-963.

6. Meier, A., P. Weidmann, M. Grimm, G. Keusch, Z. Gluck, I. Minder, and W. H. Ziegler. 1981. Pressor factors and cardiovascular pressor responsiveness in borderline hypertension. Hypertension. 3:367-372.

7. Esler, M., S. Julius, A. Zweifler, O. Randall, E. Harburg, H. Gardiner, and V. DeQuattro. 1977. Mild high-renin essential hypertension. Neurogenic human hypertension? N. Engl. J. Med. 296:405411.

8. Esler, M., S. Julius, O. S. Randall, C. N. Ellis, and T. Kashima. 1975. Relation of renin status to neurogenic vascular resistance in borderline hypertension. Am. J. Cardiol. 36:708-715.

9. Folkow, B. 1978. Cardiovascular structural adaptation; its role in the initiation and maintenance of primary hypertension. Clin. Sci. Mol. Med. 55:3s-22s.

10. Sivertsson, R. 1970. The hemodynamic importance of structural vascular changes in essential hypertension. Acta Physiol. Scand. Suppl. 343:1-56.

11. Wessels, F., and H. Zumkley. 1980. Electrolytes in blood cells. Sodium metabolism of RBC in hypertensive patients. In Intracellular Electrolytes and Arterial Hypertension, Stuttgart; Georg Thieme Verlag. 56-68.

12. Peuler, J. D., and G. A. Johnson. 1977. Simultaneous single isotope radioenzymatic assay of plasma norepinephrine, epinephrine and dopamine. Life Sci. 21:625-636.

13. Haber, E. T., T. Koerner, L. B. Page, B. Kliman, and A. Purrode. 1969. Application of a radioimmunoassay for angiotensin I to the physiologic measurement of plasma renin activity in normal healthy subjects. J. Clin. Endocrinol. Metab. 29:1349-1355.

14. Neter, J., W. Wasserman, and M. Kutaer. 1985. Applied Linear Regression Analysis. Richard D. Irwin, Inc., Homewood, Illinois, 2nd Ed.

15. Korner, P. J., M. J. West, J. Shaw, and J. B. Uther. 1974. "Steady-state" properties of the baroreceptor-heart rate reflex in essential hypertension in man. Clin. Exp. Pharmacol. Physiol. 1:65-76.

16. Goldstein, D. S., R. McCarty, R. J. Polinsky, and I. J. Kopin. 1983. Relationship between venous plasma norepinephrine and sympathetic neural activity. Hypertension. 5:552-559.

17. Esler, M., A. Zweifler, O. Randall, S. Julius, and V. DeQuattro. 
1977. Agreement among three different indices of sympathetic nervous system activity in essential hypertension. Mayo Clin. Proc. 52:379-382.

18. Wallin, G. B., G. Sundlof. 1981. G. M. Ericksson, P. Dominiak, H. Girbecker, and L. E. Lindblad. Plasma noradrenaline correlates to sympathetic nerve activity in normotensive man. Acta Physiol. Scand. III:69-73.

19. Goldstein, D. S. 1983. Plasma catecholamines in essential hypertension: An analytical review. Hypertension. 5:86-99.

20. Esler, M., G. Jennings, G. L. Jennings, J. Johns, F. Burke, P. J. Little, and P. Leonard. 1984. Estimation of 'total' renal, cardiac and splanchnic sympathetic nervous tone in essential hypertension from measurements of noradrenaline release. J. Hypertension. 2(Suppl. 3): 123-125.

21. Esler, M., G. Jennings, B. Biviano, G. Lambert, and G. Hasking. 1986. Mechanism of elevated plasma noradrenaline in the course of essential hypertension. J. Cardiol. Pharm. 8(Suppl. 5):539-543.

22. Egan, B., R. Neubig, and S. Julius. 1985. Pharmacologic reduction of sympathetic drive increases platelet alpha-2-receptor number. Clin. Pharm. Ther. 38:519-524.

23. Lockette, W., Y. Otsuka, and O. Carretero. 1986. The loss of endothelium-dependent vascular relaxation in hypertension. Hypertension. 8(Suppl. II):II62-II66.

24. Matsuda, H., E. Kuon, J. Holtz, and R. Busse. 1985. Endothelium-mediated dilations contribute to the polarity of the arterial wall in vasomotion induced by alpha-2-adrenergic agonists. J. Cardiol. Pharm. 7:680-688.
25. Cocks, T. M., and J. A. Angus. 1983. Endothelium-dependent relaxation of coronary arteries by noradrenaline and serotonin. Nature (Lond.). 305:627-630.

26. Godfraind, T., C. Eglenia, and I. A. Osachie. 1985. Role of endothelium in the contractile response of rat aorta to alpha-adrenoceptor agonists. Clin. Sci. 68(Suppl. 10):65S-71S.

27. Takeshita, A., and A. L. Mark. 1980. Decreased vasodilator capacity of forearm resistance vessels in borderline hypertension. $\mathrm{Hy}$ pertension. 2:610-616.

28. Conway, J. 1963. A vascular abnormality in hypertension. A study of blood flow in the forearm. Circulation. 27:520-529.

29. Hulthen, U. L., P. Belli, and F. R. Bühler. 1985. Vasodilator effect of nicardipine and verapamil in the forearm of hypertensive as compared with normotensive man. Br. J. Clin. Pharmacol. 20:62S$66 \mathrm{~S}$.

30. Short, D. 1966. Morphology of the intestinal arterioles in chronic human hypertension. Br. Heart J. 28:184-192.

31. Prewitt, R. L., I. I. H. Chen, and R. F. Dowell. 1982. Development of microvascular rarefaction in the spontaneously hypertensive rat. Am. J. Physiol. 243:H243-H251.

32. Harper, R. N., M. A. Moore, M. C. Marr, L. E. Watts, and P. M. Hutchins. 1978. Arteriolar rarefaction in the conjunctiva of human essential hypertensives. Microvasc. Res. 16:369.

33. Aalkjaer, C., A. M. Heagerty, M. G. Mulvany, and J. D. Swales. Studies of human resistance vessels in essential hypertension. Clin. Res. 34(2):720. (Abstr.) 